\title{
Superior Vena Cava Angiosarcoma
}

National Cancer Institute

\section{Source}

National Cancer Institute. Superior Vena Cava Angiosarcoma. NCI Thesaurus. Code C5378.

A malignant vascular neoplasm arising from the superior vena cava. 